\title{
Recent advances in our understanding of risk-sensitive foraging preferences
}

\author{
Melissa Bateson \\ Evolution and Behaviour Research Group, School of Biology, University of Newcastle, Henry Wellcome Building for \\ Neuroecology, Framlington Place, Newcastle upon Tyne, NE2 4HH, UK
}

\begin{abstract}
Many experiments have shown that foraging animals are sensitive to the riskiness, or variance, associated with alternative food sources. For example, when offered a choice of a constant feeding option that always offers three seeds, and a risky option that offers either no seeds or six seeds with equal probability, most animals tested will be either risk-averse or risk-prone, preferring either the fixed or variable option respectively. Whether animals are risk-averse or risk-prone appears to depend on a range of factors, including the energetic status of the forager, the type of variance associated with the feeding options and even the number of feeding options between which the animal is choosing. These behavioural phenomena have attracted much theoretical interest, and a range of different explanations have been suggested, some based on a consideration of the psychological mechanisms involved in decision making, and others on a consideration of the Darwinian fitness consequences of risk-averse or risk-prone behaviour for the forager. A brief review of the recent literature on risk-sensitive foraging will be presented, focusing on results from the two experimental systems with which I have been involved: wild rufous hummingbirds (Selasphorus rufus) foraging on artificial flowers; European starlings (Sturnus vulgaris) foraging in operant boxes in the laboratory. It will be argued that to understand the foraging decisions of animals account needs to be taken of both the psychological mechanisms underlying decisionmaking and the fitness consequences of different decisions for the forager.
\end{abstract}

Risk-sensitive foraging: Energy-budget rule: Risk-averse: Risk-prone

For many small animals, making the right foraging decisions on a minute-by-minute basis can literally make the difference between life and death. For example, every spring, $3.5 \mathrm{~g}$ rufous hummingbirds (Selasphorus rufus) make the journey from Mexico to their breeding grounds $1400 \mathrm{~m}$ up in the Canadian Rocky Mountains. The birds arrive in May when freezing night-time temperatures and heavy snowfalls are still not unusual. In order to supply their energetic needs they feed every $10-15 \mathrm{~min}$ from dawn until dusk, and when night comes they have to drop their metabolic rate and go into torpor until they are able to resume foraging again in the morning. A bird that makes just a few poor foraging decisions could easily be left with insufficient energy reserves to survive until morning, especially if the night is cold, or snowfall delays the resumption of foraging. Given the very real possibility of starving to death, there are strong reasons to believe that natural selection acts extremely strongly on the foraging behaviour of these birds. Although not all birds are as energetically close to the edge as hummingbirds, due to their relatively high metabolic rates and small body size, we would expect the foraging behaviour of all birds to have been optimised by natural selection.

Since the seminal papers of Charnov $(1976 a, b)$ first published 25 years ago, the field of Optimal Foraging Theory has been dedicated to understanding the ways in which natural selection has optimised foraging behaviour. Within this field my own research has focused on the problem of how animals evaluate alternative feeding options and make decisions between them. The aim here is to review how such foraging decisions are affected by the variance, or riskiness, in the pay-offs obtained from alternative foraging options. A very brief summary of the field of risk-sensitive foraging will be presented, followed by more in-depth descriptions and discussion of some recent findings in the two experimental systems with which I have been involved, i.e. wild rufous hummingbirds foraging on artificial flowers, and European starlings (Sturnus vulgaris) foraging in operant boxes in the laboratory. 
Risk can only be a factor in foraging decisions if at the time of making a decision the forager cannot precisely determine the pay-offs available from alternative options. It makes sense, therefore, to study sensitivity to risk in species for which this situation is likely to be common. There are aspects of the ecology of both hummingbirds and starlings that make them ideal candidates for the study of risk sensitivity. In the case of the hummingbirds, a large proportion of the birds' food intake comes from nectar obtained from flowers. The nectar is hidden within the flower, and it is impossible for the bird to determine before visiting the flower exactly how much nectar will be available. Variation in the nectar content within the flowers of a single species, due to variation in nectar production by the plant and previous exploitation of the flower by other foragers, means that nectar is a naturally risky resource. Similarly, starlings spend much of the year feeding on invertebrate larvae hidden beneath the surface of the ground. The larvae are distributed in patches, and exploitation by other starlings will have caused depletion in some areas, again creating a situation where a bird cannot precisely predict the pay-off that will be obtained by visiting a particular area. Thus, both hummingbirds and starlings are species likely to have encountered foraging decisions involving risk during their evolutionary history, and it is thus reasonable to assume that they may have evolved behavioural strategies for dealing with risk.

\section{Long-term rate of energy intake}

The optimality approach to understanding adaptation requires us to identify the currency that foraging animals are maximising (for a review, see Stephens \& Krebs, 1986). It is accepted that ultimately this currency must be Darwinian fitness; however, in the short term animals need proxies for fitness that can be quickly computed and maximised on a minute-by-minute basis. In the first generation of optimal foraging models the currency assumed was what is known as long-term rate of energy intake, which is equal to the expected amount of energy obtained from a given foraging option divided by the expected total length of time spent obtaining this energy:

$$
\text { Long-term rate }=\frac{E(\text { Amount })}{E(\text { Time })}
$$

Long-term rate is assumed to correlate well with fitness, because an animal maximising this currency maximises the energy it obtains per unit time spent foraging, thus maximising the amount of time left free for other fitness-enhancing activities such as reproduction.

A clear prediction arising from long-term rate maximising is that animals should not be sensitive to the variance, or risk, associated with a foraging option. This prediction occurs because, in order to compute the long-term rate associated with a foraging option, it is assumed that the animal computes the ratio of the total amount of food obtained from the option to the total amount of time spent obtaining it. Thus, any variation in either the amount of food or time is lost, and an option that provides 4 units of food with certainty should be evaluated as equivalent to an option that provides either 2 or 6 units of food on each visit with equal probability.

Since the energetic value and handling time associated with a particular foraging option are usually immutable properties of that option, long-term rate of energy intake is defined by economists as an 'absolute' currency. If options are evaluated using any absolute currency, then certain predictions follow. Decision-making based on maximising the value of an absolute currency should always result in rational behaviour, including transitivity and regularity (Tversky \& Simonson, 1993).

Long-term rate maximising has had some notable successes in explaining foraging decisions in animals (for example, see Kacelnik, 1984). However, there is now mounting evidence that animal decision making is considerably more complex. Many experiments have shown that foraging animals are sensitive to the riskiness (variance) associated with alternative food sources (for reviews, see Kacelnik \& Bateson, 1996; Bateson \& Kacelnik, 1998), and there is a growing body of evidence that foraging decisions may sometimes be intransitive (Shafir, 1994; Waite, 2001) or irregular (Hurly \& Oseen, 1999; Bateson et al. 2002; Shafir et al. 2002), suggesting that animals may not be using an absolute currency to evaluate alternative options. In order to understand these findings we need to consider both the evolutionary pressures with which animals are faced, and also how mechanistic constraints may influence how an adaptive decision rule is actually implemented.

\section{Risk sensitivity}

In a typical experiment to test for risk sensitivity an animal is presented with a simultaneous choice of two foraging options that differ in riskiness. The most usual comparison is between a constant option with zero variance, for which the bird can learn exactly how much food it will obtain and how much time it will take it to get the food, and a variable option, in which the amount of food or the delay associated with obtaining food is picked from a probability distribution with a mean equal to the value in the constant option, but a non-zero variance. If an animal is insensitive to risk it should be indifferent between two options with the same mean, whereas a risk-averse animal will show a preference for the constant option and a risk-prone animal will show preference for the more variable option.

There is a large body of empirical data showing that foraging animals are sensitive to risk. In an extensive review of this literature Kacelnik \& Bateson (1996) analysed the results from fifty-nine studies spanning twenty-eight species, including insects, fish, birds and mammals. Risksensitive preferences were observed in all these taxonomic groups and in the majority of studies, suggesting that risk sensitivity is both a common and taxonomically-widespread phenomenon. Some clear patterns have emerged from this extensive literature. First, there is a strong suggestion that the direction of risk-sensitive preferences may be influenced by the energetic status of the forager, with animals on positive energy budgets tending to be risk-averse and animals on negative energy budgets tending to be riskprone. Second, there is clear evidence that the direction of risk-sensitive preferences is also affected by whether the 
variance is programmed in the amount of food obtained by the forager, or in the time delay associated with acquiring the food. When variance is in amount, animals are more usually risk-averse, whereas when variance is in delay, animals are almost universally risk-prone. Finally, since the publication of the Kalcenik \& Bateson (1996) review, a couple of recent papers have also suggested that both the direction and magnitude of risk-sensitive preferences may be influenced by the number of options available in the choice set (Hurly \& Oseen, 1999; Bateson, 2002). Each of these three findings will be discussed in turn, and some of the explanations put forward to explain them will be described.

\section{Energy-budget effects}

The clearest demonstration of the effect of energy budget on risk sensitivity comes from a study of the effects of ambient temperature on the foraging decisions of yellow-eyed juncos (Junco phaeonotus; small North American passerine birds). The birds were presented with a choice between a constant foraging option that always provided three seeds and a variable option that provided either no seeds or six seeds with equal probability. The experiment was carried out at two temperatures: $1^{\circ} \mathrm{C}$, at which the birds were calculated to be on a negative energy budget (i.e. the average reward of three seeds per trial was not enough to maintain their body weights); $19^{\circ} \mathrm{C}$, at which the birds were calculated to be on a positive energy budget (i.e. the average reward was sufficient for their needs). The birds' preferences for the constant and variable foraging options depended on the temperature: at the low temperature they were risk-prone; at the higher temperature they were risk-averse.

An adaptive explanation for this result was first provided by Stephens (1981). He suggested that in the case of a small bird, such as a hummingbird, faced with a single foraging decision to go before a long cold night, the difference between a constant foraging option with a known outcome and a risky option could be critical. If the pay-off from the constant option is enough to provide the bird with sufficient energetic reserves to survive the night (i.e. the bird is on a positive energy budget), then it is optimal for it to choose this option and not take the risk that it will obtain nothing from the variable option. However, if pay-off from the constant option is insufficient for the bird to survive the night (i.e. it is on a negative energy budget), then its only chance of survival is to choose the variable option and hope to obtain the larger pay-off. It is optimal to choose the variable option because although it only provides a $50 \%$ chance of survival, choosing the constant option will result in certain death. The latter pattern of behaviour is summarised in the 'energy-budget rule', which states that for an animal on a positive energy budget it is optimal to be risk-averse, whereas for an animal on a negative energy budget it is optimal to be risk-prone.

The energy-budget rule produces a selective advantage for sensitivity to variance by assuming a non-linear relationship between rate of energy intake and fitness (McNamara \& Houston, 1992). This non-linearity is achieved by introducing a threshold level of energy reserves below which the forager will die (for example, see Stephens,
1981; McNamara et al. 1991). A graphical representation of the energy-budget model with a single survival threshold is shown in Fig. 1. The three curves show the probability distributions of pay-offs of different sizes provided by low-, intermediate- and high-variance foraging options, all of which provide the same mean rate of energy intake. Also shown is the probability of dying of starvation if the forager chooses a given option. Fig. 1(A) demonstrates that for an animal on a positive energy budget (i.e. the threshold is to the left of the mean rate available), it is always optimal to be risk-averse, since the lower the variance in rate of intake the lower the probability of starvation. In contrast, Fig. 1(B) shows the situation for an animal on a negative energy budget (i.e. the survival threshold is to the right of the mean rate of energy intake available). In this case it is always optimal to be risk-prone, since the higher the variance in rate of intake the higher the probability of survival. Reducing ambient temperature should have the effect of shifting the survival threshold to the right, since at lower temperatures more energy is needed for survival. Thus, this model predicts that animals should be risk-averse at higher temperatures and risk-prone at lower temperatures. This relationship has been reported by Caraco et al. (1990).

Despite the appeal of the energy-budget model, widespread empirical support for it has been patchy (for review see Bateson \& Kacelnik, 1998). In starlings Reboreda \& Kacelnik (1991) reported that birds that were less efficient at utilising the food hopper, and as a consequence got smaller food rewards, were more risk-prone than more efficient birds. This observation agrees with the predictions of the energy-budget rule. However, since the amount of food the birds obtained was not manipulated the evidence is only correlational. Two attempts to experimentally manipulate food intake in starlings have both failed to find budget effects on risk-sensitive preferences (Bateson \& Kacelnik, 1997; Brito-e-Abreu \& Kacelnik, 1999).

\section{Risk in amount $v$. risk in time}

A second problem with the energy-budget account described earlier is that it does not obviously explain why there should be such great difference in the way animals respond to variability in amount of food and delay to food. Explanations for this phenomenon have come from thinking about the psychological mechanisms of choice. At least two different mechanistic explanations have been suggested for the difference in response to variability in amount of food and time (for a review of the different models, see Kacelnik \& Bateson, 1997; Bateson \& Kacelnik, 1998). However, here the focus will be on the explanation that comes from considering the details of the rate currency used to evaluate alternative options.

As described earlier, risk-sensitive foraging experiments are usually designed such that the long-term rate of energy available from the constant and variable options is equal. The rationale for equalising long-term rate is that if animals are risk-sensitive when presented with constant and variable options of equal long-term rate, then they must be sensitive to the variance in their rate of intake, since this factor represents the only difference between the two options. However, this conclusion only holds if long-term rate of 
(A)

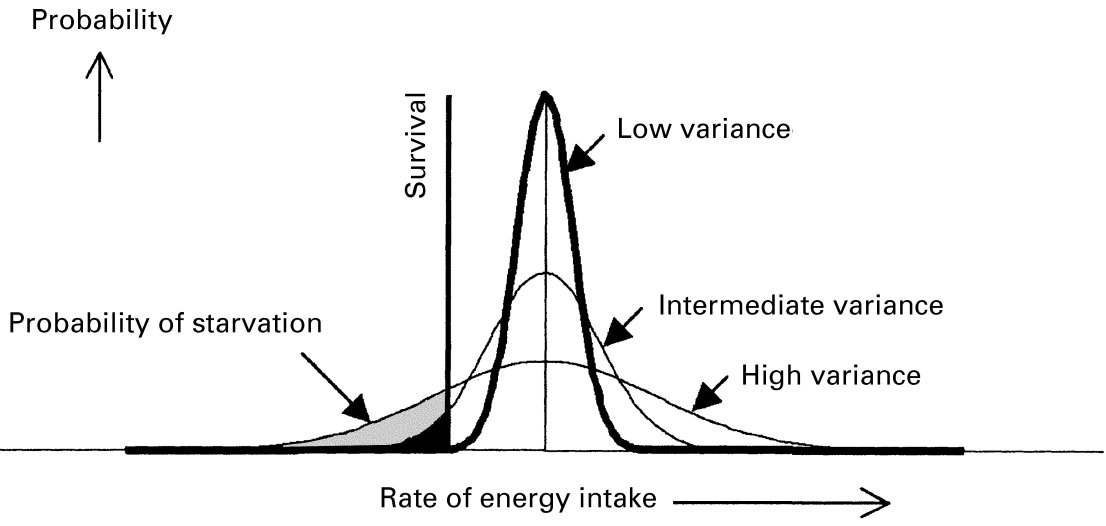

(B)

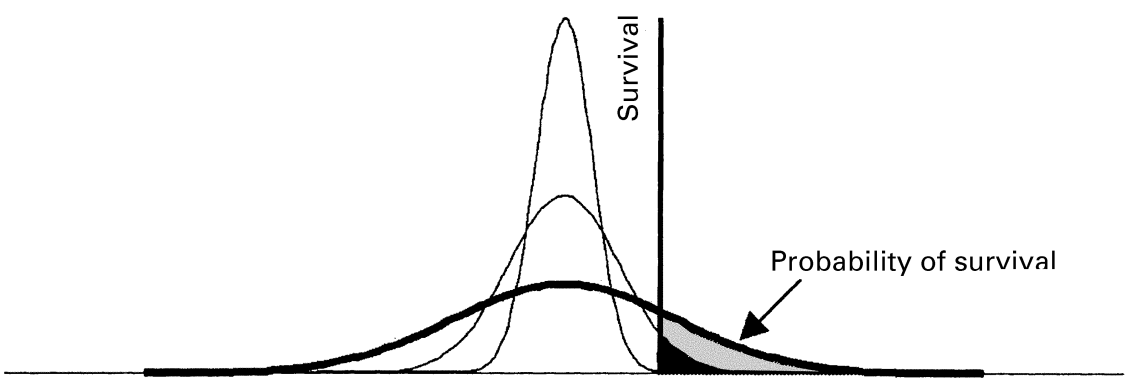

Fig. 1. The energy-budget rule demonstrated graphically with three alternative foraging options differing in the extent of variance in rate of energy intake (low, intermediate or high). Note that all three options have the same mean rate of energy intake associated with them. (A), The starvation threshold is below the mean rate of intake provided by the three options (i.e. a positive energy-budget situation), and it is thus optimal to be risk-averse, preferring the low-variance option, because this option minimises the probability of starvation. (B), Conversely, the starvation threshold (I) is above the mean rate of intake provided by the three options (i.e a negative energy-budget situation), and it is thus optimal to be risk-prone, preferring the high-variance option, because this option maximises the probability of survival. (-), The optimal option to choose in both A and B.

energy intake is the basic currency maximised by foraging animals. This latter assumption has been the subject of lively debate, because some empirical evidence appears to favour an alternative rate currency known as short-term rate (Harder \& Real, 1987; Possingham et al. 1990). Short-term rate is defined as the ratio of the expected value for amount of food to the time taken to acquire it:

$$
\text { Short term rate }=\mathrm{E} \frac{\text { Amount }}{\text { Time }}
$$

Long- and short-term rates both involve computing the ratio of the amount of food gained to the length of time taken, but the two currencies differ in the position at which it assumed the animal computes an average, or expectation. Consider an animal that is faced with computing the value of a variable foraging patch that provides either no seeds or six seeds on each visit with equal probability. Computing the long-term rate associated with the patch involves computing an expectation of the number of seeds that will be obtained on a single visit (in this case three) and dividing this number by the expectation of how long it takes to acquire and consume these seeds. In contrast, computing short-term rate involves computing the number of seeds obtained divided by the time taken each time the patch is visited, and then calculating an expectation of the overall rate associated with the option by averaging these individual rates.

Long- and short-term rates are equivalent if there is no variance in the time associated with acquiring food; however, when there is variance in time, long- and shortterm rates diverge (Caraco et al. 1992). The difference between long- and short-term rates depends on the source of variance in the variable option. If long-term rates are equalised in the constant and variable option, then a variable-delay option, in which the time delay associated with acquiring food is varied, will provide a higher shortterm rate of intake than a constant option. Conversely, a variable-amount option, in which the amount of food is varied, will provide a lower short-term rate of intake than a constant option. The effect of variability in amount on shortterm rate occurs because there is usually a positive correlation between the amount of food received and the time taken to consume this food. Thus, varying the amount of food causes variance in time, and as a consequence of this correlation, differences in short-term rate.

A special case occurs if there is no correlation between the amount of food and the time taken to consume it. In this situation the short-term rate for a variable-amount option 
does not differ from the long-term rate. Such a dissociation between amount of food and consumption time is likely to occur if the amount of food is manipulated by changing the concentration of a sugar solution, because for most nectarivores, increases in nectar concentration do not cause large correlated increases in consumption time.

These properties of short-term rate have led to the prediction that foragers should respond differently to risk, depending on its source. Specifically, animals should be risk-averse when variance is in the amount of food gained, but risk-prone when variance is in the time associated with acquiring the food. This predicted pattern is seen both across species and studies, and also within studies that have examined the effects of manipulating both types of variance in the same subjects. For example, a number of studies have investigated risk-sensitive foraging in European starlings trained on operant tasks to forage for food by pecking at illuminated keys. In a typical choice test the bird is presented with two options, one of which offers a fixed amount of food after a fixed amount of time, while the other is variable, either in the time delay to obtain the food or in the amount of food delivered to the bird. In such experiments starlings are risk-averse when variance is in the number of food pellets delivered or the length of access to a food hopper, but risk-prone when variance is in the time delay associated with acquiring food (Reboreda \& Kacelnik, 1991; Bateson \& Kacelnik, 1995).

In the case of the latter result, support for short-term rate maximising has been quantitative as well as qualitative. Theoretically, if the risk proneness observed in a risksensitivity experiment with variable delay is due to shortterm rate maximising, then it is possible to compute the magnitude by which it should be necessary to reduce the delay to food in the constant option in order for the shortterm rates in the constant and variable options to be equated, and thus for the starlings to become indifferent between the two options. It can be shown that this indifference point occurs when the delay in the constant option is equal to the harmonic mean (i.e. the reciprocal of the mean of the reciprocals) of the two possible delays in the variable option (Bateson \& Kacelnik, 1996). We tested this prediction in European starlings by first establishing risk proneness in a choice between a constant- and variable-delay option, and then titrating the delay to food in the constant option until the starlings became indifferent between the two options (Bateson \& Kacelnik, 1996). The value of the delay in the constant option at indifference was not significantly different from the harmonic mean of the two delays in the variable option, thus providing strong quantitative support for the short-term rate maximising hypothesis for risk sensitivity.

Risk aversion to variance in amount is only predicted where there is a correlation between energy obtained and consumption time, whereas indifference to variance in amount of food is predicted if no such correlation exists. In support of this prediction, bumblebees (Bombus edwardsii) and bananaquits (Coereba flaveola) are riskaverse to variance in nectar volume, but indifferent to variance in nectar concentration, but this difference is yet to be demonstrated in a single balanced experiment (Real, 1981; Waddington et al. 1981; Real et al. 1982; Wunderle \& O’Brien, 1985; Waddington, 1995).

In a recent experiment on risk-sensitive foraging in rufous hummingbirds we set out to test the prediction that nectarivores should be more risk-averse to variance in nectar volume than to variance in nectar concentration (M Bateson, SD Healy and TA Hurly, unpublished results). We examined the preferences of twelve wild rufous hummingbirds presented with binary choices between artificial flowers differing in either variance in nectar volume (volume treatment) or variance in nectar concentration (concentration treatment). The birds chose between two boards of artificial flowers: a constant board that comprised sixteen flowers containing $15 \mu \mathrm{l}$ sucrose solution $(20 \%$, $\mathrm{w} / \mathrm{v})$, and a variable board that differed between treatments. In the volume treatment the variable board comprised twelve flowers containing $5 \mu \mathrm{l}$ sucrose $(20 \%$, w/v) and four flowers containing $45 \mu \mathrm{l}$ sucrose $(20 \%$, w/v), whereas in the concentration treatment the variable board comprised twelve flowers containing $15 \mu \mathrm{l}$ sucrose $(9.82 \%, \mathrm{w} / \mathrm{v})$ and four flowers containing $15 \mu \mathrm{l}$ sucrose $(50.55 \%$, w/v). We calculated the short-term net rate of energy intake associated with each of the flower types, and maximisation of short-term rate led to the prediction that the birds should be risk-averse in the volume treatment but risk-prone in the concentration treatment. However, contrary to these predictions the birds were risk-indifferent in the volume treatment and risk-averse in the concentration treatment. Thus, overall, they were more risk-averse in the concentration treatment than the volume treatment, which was the exact opposite of what we predicted. At the moment we do not have a satisfactory explanation for this difference. Longterm rate maximising does not explain the difference between the volume and concentration treatments, because all three flower types were designed to provide identical long-term rates of energy intake. A recent analysis of the published literature on risk sensitivity suggests that the magnitude of risk-averse preferences in necatrivores can be explained by the $\mathrm{CV}$ in the variable option, with greater variability resulting in greater risk aversion. However, since the two variable flower types in our experiment were also designed to have equal $\mathrm{CV}$ in the variable quantity, this explanation is also not adequate to explain the difference we observed. At present, our only explanation for the treatment difference is that perhaps risk aversion was more extreme in the concentration treatment because different nectar concentrations may be easier to perceive than different nectar volumes.

Thus, to summarise this section, current evidence for the short-term rate maximising account of risk sensitivity is equivocal. The hypothesis works well in explaining risk proneness to variability in delay to food, but at present it does not seem to help explain risk aversion to variance in the amount of food. These latter results add to the mounting evidence that animals not only treat risk in amount and delay differently, but that we also may need to consider different types of explanation for the two kinds of risk. 


\section{Binary $v$. trinary choices}

Until very recently, all experimental studies of risk-sensitive foraging have employed binary choices in which an animal is required to choose between two options differing in variance. However, recent results from both rufous hummingbirds and European starlings have raised the possibility that in focusing only on binary choice we may have been missing some interesting aspects of risk-sensitive foraging.

The first suggestion that this may be the case comes from a study of rufous hummingbirds in which Hurly \& Oseen (1999) investigated the choices of the birds for three artificial flower types: a constant flower that always provided $30 \mu \mathrm{l}$ nectar $(20 \%, \mathrm{w} / \mathrm{v})$; a low-variance flower that gave 20 or $40 \mu 1$ with equal probability; a high-variance flower that gave either 10 or $40 \mu \mathrm{l}$ with equal probability. Their experiment differed from earlier studies of risk because, in addition to making the usual binary comparisons between the constant and variable flowers, they also gave the birds a simultaneous trinary choice between all three flower types. In the two binary tests the birds were riskaverse, preferring the constant option in both comparisons. However, in the trinary test they reported the unexpected result that the birds switched to preferring the low-variance option to both the constant and high-variance options. Two aspects of this result were novel: first, the switch in preference between binary and trinary treatments; second, the preference for an intermediate level of variance in the trinary-choice treatment. Each of these findings will be considered in turn.

The switch in preference arising from an increase in the proportion of choices allocated to the low-variance option in the trinary treatment as compared with the binary (constant $v$. low-variance) treatment constitutes a violation of regularity. A violation of regularity occurs when the proportion of choices for a particular option increases when an additional option is added to the choice set; regularity in choice is commonly regarded as a hallmark of rational choice. This finding has important consequence for the mechanisms of choice employed by the hummingbirds, because it suggests that they do not use an absolute currency to evaluate alternative options (Tversky \& Simonson, 1993). Since long-term rate, short-term rate and variance are all absolute currencies, the result obtained by Hurly \& Oseen (1999) suggests that none of the existing explanations for risk-sensitive preferences can be complete. Violations of regularity can only be obtained if the value assigned to a particular option is not an absolute quantity, but instead is computed relative to the other options currently available. Such comparative currencies have been suggested to explain violations of regularity in human choice behaviour, but this study provides the first conclusive evidence that we may need to consider similar mechanisms in the context of animal choice.

The preference for the intermediate low-variance option observed in the trinary treatment is interesting, because it is also not predicted by any of the current explanations for risk sensitivity. The energy-budget rule predicts that animals should prefer the constant option if they are on a positive energy budget, but the high-variance option if they are on a negative budget. Similarly, the short-term rate approach also predicts a monotonic relationship between variance and risk sensitivity, because the greater the variance in volume of nectar associated with a foraging option the lower the estimate of short-term rate for that option.

In order to establish whether species other than rufous hummingbirds might be sensitive to the size of the choice set when making risk-sensitive choices, the study of Hurly \& Oseen (1999) was replicated in European starlings foraging in operant chambers in the laboratory (Bateson, 2002). Analogous with the Hurly \& Oseen (1999) study the starlings preferences were analysed for three foraging options differing in the variance in number of pellets provided: a constant option that provided a fixed number of food pellets; a low-variance option with a CV of $71 \%$ in the number of pellets, and a high-variance option with a CV of $106 \%$ in the number of pellets. The preferences of the birds were tested in three binary treatments (constant $v$. low-variance, constant $v$. high-variance and low-variance $v$. high-variance) and one trinary treatment (constant $v$. low-variance $v$. high-variance). The results were not as striking as those of Hurly \& Oseen (1999) because the birds varied in their preferences, some preferring the constant option and some the low-variance option. However, overall there was an effect of set size, with the birds increasing their relative preference for their own preferred option in the trinary treatment. In the trinary treatment seven of eight birds had a clear ranking of the three options, with four birds preferring the low-variance option and three birds preferring the constant option. Additionally, the strength of individual birds' preferences for the lowvariance option over the constant option in the trinary treatment was positively related to the temperature in the aviary during this treatment, with the birds becoming more risk-averse (i.e. higher preference for the constant option) the lower the ambient temperature. Note that this effect of temperature on preference is in the opposite direction to that predicted by the energy-budget rule.

\section{Twin-threshold model}

The single-threshold energy-budget rule described earlier cannot explain two features of the results from the trinary choice tests in hummingbirds and starlings: first, the preference for an intermediate variance option displayed by both species; second, the increasing risk aversion with decreasing temperature displayed by the starlings. However, TA Hurly (unpublished results) has recently investigated the consequences of adding a second energetic threshold to the basic energy-budget model. Fig. 2(A) shows the effect of adding an additional higher energetic threshold to the model. The threshold is labelled 'reproduction' and could, for instance, represent an energy threshold above which reproduction is possible. In the situation shown, the intermediate-variance option could be optimal for an iteroparous animal, for which it is more important to survive than to reproduce at any point in time, because the high-variance option gives some probability of starvation and the low-variance option gives zero probability of reproduction, whereas the intermediatevariance option give zero probability of starvation but some probability of reproduction. Fig. 2(B) shows the effect of 
(A)

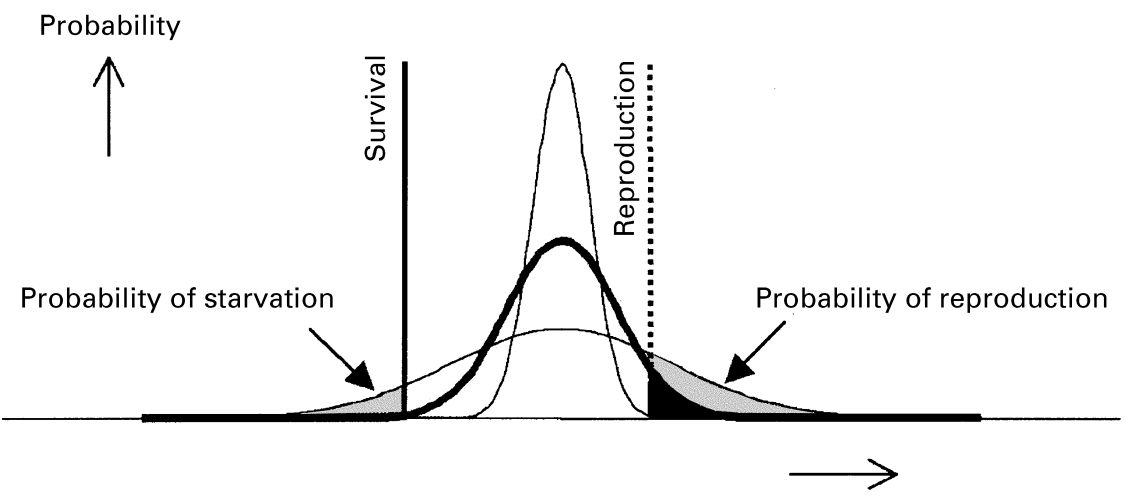

(B)

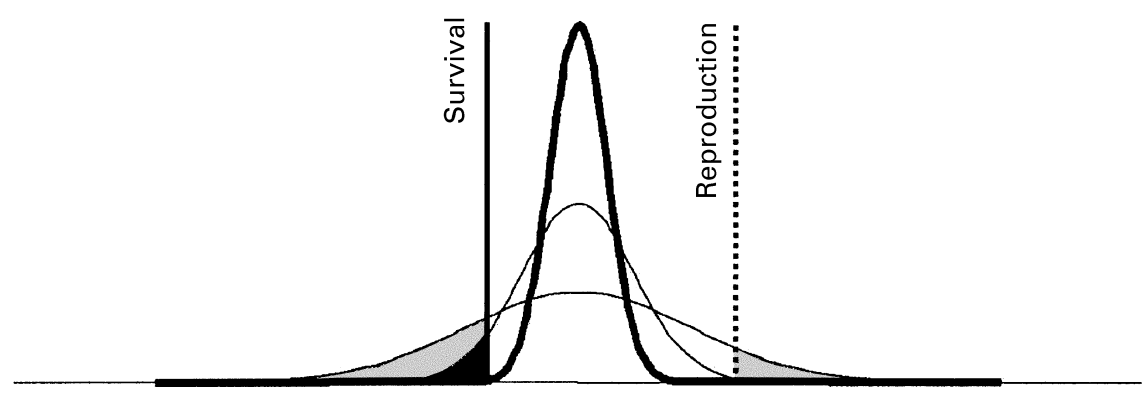

Fig. 2. A graphical representation of the twin-threshold model with the same three alternative foraging options represented in Fig.1 (low, intermediate and high variance in rate of energy intake) (I), The starvation threshold; (I), reproduction threshold. (A), A situation with a higher ambient temperature than $B$, because both thresholds are shifted to the right in $B$. In $A$ it is optimal to prefer the intermediate-variance option, because this option maximises the probability of reproduction with no reduction in the probability of survival. However, in B it is optimal to be risk-averse and prefer the low-variance option, because this option minimises the probability of starvation. (-), The optimal option to choose in both $A$ and $B$.

reducing the ambient temperature, which, as in Fig. 1, should result in shifting both thresholds to the right. In this situation the low-variance option has become optimal, because both high and low variance offer some probability of starvation. Thus, adding a second threshold to the model reverses the predictions of the single-threshold model and predicts that animals should be more risk averse at lower temperatures.

The twin-threshold model therefore predicts two aspects of the results obtained: first, the preference for the intermediate-variance option; second, the positive correlation between temperature and preference for the intermediatevariance option over the low-variance option observed in starlings. The model also makes a number of additional predictions, which TA Hurly (unpublished results) has started to test in hummingbirds. For example, the model predicts that if the mean volume of nectar provided by the three options is reduced, then the birds should increase their preference for the low-variance option, and when it is shifted higher they should increase their preference for the highvariance option. He recently confirmed these predictions in hummingbirds (TA Hurly, unpublished results).

\section{Conclusions}

It should be clear from the present brief review that, while we are continuing to find out more about the various factors that influence risk-sensitive foraging decisions, we are still a long way from a unified explanation for the various phenomena that have been described. In fact, from the evidence presented in the present review, it is starting to look as if risk sensitivity to variance in amount and delay may have entirely different explanations. The models that work well for variance in amount, such as the energy budget-rule, appear not to work for variance in delay and, conversely, the models that work for variance in delay, such as short-term rate maximising, do not seem to explain responses to variance in amount.

The recent results, showing that for variability in amount at least, the magnitude and even direction of risk-sensitive preferences are influenced by the size of the choice set, are extremely worrying for all existing models of risk-sensitive foraging. These results suggest that models of risk-sensitive foraging derived from results from binary experiments may not generalise to choice sets with more than two alternative 
options. More importantly, the results suggest that we may have to abandon the idea that animals evaluate alternative options using any absolute currency, but instead evaluate options comparatively, as has been found in human consumers (for example, see Huber et al. 1982; Wedell, 1991).

\section{Acknowledgements}

I am funded by a University Research Fellowship from the Royal Society, and receive additional financial support from my Department, NERC and the Wellcome Trust. I am grateful to Andy Hurly for sharing his twin-threshold model with me, and to the Nutrition Society for inviting me to speak at their meeting on Perspectives in the study of food intake.

\section{References}

Bateson M (2002) Context-dependent foraging preferences in risk sensitive starlings. Animal Behaviour (In the Press).

Bateson M, Healy SD \& Hurly TA (2002) Irrational choices in hummingbird foraging behaviour. Animal Behaviour 63, 587-596.

Bateson M \& Kacelnik A (1995) Preferences for fixed and variable food sources: variability in amount and delay. Journal of the Experimental Analysis of Behaviour 63, 313-329.

Bateson M \& Kacelnik A (1996) Rate currencies and the foraging starling: the fallacy of the averages revisited. Behavioral Ecology 7, 341-352.

Bateson M \& Kacelnik A (1997) Starlings' preferences for predictable and unpredictable delays to food. Animal Behaviour 53, 1129-1142.

Bateson M \& Kacelnik A (1998) Risk-sensitive foraging: decision making in variable environments. In Cognitive Ecology, pp. 297-341 [R Dukas, editor]. Chicago, IL: Chicago University Press.

Brito-e-Abreu F \& Kacelnik A (1999) Energy budgets and risksensitive foraging in starlings. Behavioral Ecology 10, 338-345.

Caraco T, Blanckenhorn WU, Gregory GM, Newman JA, Recer GM \& Zwicker SM (1990) Risk-sensitivity: ambient temperature affects foraging choice. Animal Behaviour 39, 338-345.

Caraco T, Kacelnik A, Mesnik N \& Smulewitz M (1992) Shortterm rate maximization when rewards and delays covary. Animal Behaviour 44, 441-447.

Charnov EL (1976a) Optimal foraging: attack strategy of a mantid. American Naturalist 110, 141-151.

Charnov EL (1976b) Optimal foraging: the marginal value theorem. Theoretical Population Biology 9, 129-136.

Harder L \& Real LA (1987) Why are bumble bees risk-averse? Ecology 68, 1104-1108.

Huber J, Payne JW \& Puto C (1982) Adding asymmetrically dominated alternatives: violations of regularity and the similarity hypothesis. Journal of Consumer Research 9, 89-98.
Hurly TA \& Oseen MD (1999) Context-dependent, risk-senstive foraging preferences in wild rufous hummingbirds. Animal Behaviour 58, 59-66.

Kacelnik A (1984) Central place foraging in starlings (Sturnus vulgaris) I. Patch residence time. Journal of Animal Ecology 53, 283-299.

Kacelnik A \& Bateson M (1996) Risky theories - the effects of variance on foraging decisions. American Zoologist 36, 402-434.

Kacelnik A \& Bateson M (1997) Risk sensitivity: cross-roads for theories of decision-making. Trends in Cognitive Sciences 1, 304-309.

McNamara JM \& Houston AI (1992) Risk-sensitive foraging: a review of the theory. Bulletin of Mathematical Biology 54, $355-378$

McNamara J, Merad S \& Houston AI (1991) A model of risksensitive foraging for a reproducing animal. Animal Behaviour 41, 787-792.

Possingham HP, Houston AI \& McNamara JM (1990) Risk-averse foraging in bees: a comment on the model of Harder and Real. Ecology 71, 1622-1624.

Real LA (1981) Uncertainty and pollinator-plant interactions: the foraging behavior of bees and wasps on artificial flowers. Ecology 62, 20-26.

Real LA, Ott J \& Silverfine E (1982) On the tradeoff between mean and variance in foraging: an experimental analysis with bumblebees. Ecology 63, 1617-1623.

Reboreda JC \& Kacelnik A (1991) Risk sensitivity in starlings: variability in food amount and food delay. Behavioral Ecology 2 , 301-308.

Shafir S (1994) Intransitivity of preferences in honey bees: support for 'comparative' evaluation of foraging options. Animal Behaviour 48, 55-67.

Shafir S, Waite TA \& Smith BH (2002) Context-dependent violations of rational choice in honeybees (Apis mellifera) and gray jays (Perisoreus canadensis). Behavioral Ecology and Sociobiology 51, 180-187.

Stephens DW (1981) The logic of risk-sensitive foraging preferences. Animal Behaviour 29, 628-629.

Stephens DW \& Krebs JR (1986) Foraging Theory. Princeton, NJ: Princeton University Press.

Tversky A \& Simonson I (1993) Context-dependent preferences. Management Science 39, 1179-1189.

Waddington KD (1995) Bumblebees do not respond to variance in nectar concentration. Ethology 101, 33-38.

Waddington KD, Allen T \& Heinrich B (1981) Floral preferences of bumblebees (Bombus edwardsii) in relation to intermittent versus continuous rewards. Animal Behaviour 29, 779-784.

Waite TA (2001) Intransitive preferences in hoarding gray jays (Perisoreus canadensis). Behavioral Ecology and Sociobiology 50, 116-121.

Wedell D (1991) Distinguishing among models of contextually induced preference reversals. Journal of Experimental Psychology 17, 767-778.

Wunderle JM \& O'Brien TG (1985) Risk-aversion in hand reared bananaquits. Behavioral Ecology and Sociobiology 17, 371380 . 